\title{
Resolução de um Problema de Despacho Econômico de Carga utilizando Enxames de Partículas e Vaga-lumes
}

\author{
Jefferson Oliveira dos Santos ${ }^{1}$ \\ Centro de Tecnologia de Alegrete, UNIPAMPA, Alegrete, RS \\ Kléber Kapelinski ${ }^{2}$ \\ Centro de Tecnologia de Alegrete, UNIPAMPA, Alegrete, RS \\ Eduardo Machado dos Santos ${ }^{3}$ \\ Centro de Tecnologia de Alegrete, UNIPAMPA, Alegrete, RS \\ João Plínio Juchem Neto ${ }^{4}$ \\ Centro de Tecnologia de Alegrete, UNIPAMPA, Alegrete, RS
}

\begin{abstract}
Resumo. O objetivo deste trabalho é resolver um problema de despacho econômico de carga considerando quinze unidades geradoras termoelétricas, perdas nas linhas de transmissão e limites máximos e mínimos de geração para cada geradora. Para tanto, serão utilizadas as heurísticas otimização por enxame de partículas (PSO) e de vaga-lumes (FA), e os resultados comparados com a solução obtida via método dos multiplicadores de Lagrange (MML). Como principal resultado, obteve-se que a melhor solução obtida pelo FA foi muito mais precisa do que a obtida pelo PSO, quando comparados com o MML, embora a um custo computacional consideravelmente maior.
\end{abstract}

Palavras-chave. Despacho Econômico de Carga, Otimização por Enxame de Partículas, Otimização por Enxame de Vaga-lumes, Multiplicadores de Lagrange.

\section{Introdução}

O despacho econômico de carga (DE) consiste em um problema de otimização restrito não-linear onde se deseja alocar a carga demandada pelo sistema elétrico de potência nas unidades geradoras, de modo a alcançar o menor custo total de geração [3]. Quando o sistema analisado possui muitas unidades geradoras, geralmente se torna muito trabalhoso resolvê-lo utilizando o método tradicional dos multiplicadores de Lagrange (MML) [7]. Nestes casos, estão sendo muito utilizados heurísticas bioinspiradas, que apresentam como diferencial sua fácil implementação e eficiência $[4,6]$. Heurísticas bastante utilizadas na área de Engenharia são a otimização por enxame de partículas (PSO) [4] e otimização por enxame de vaga-lumes (AF) [8].

\footnotetext{
${ }_{1}$ jeffersonoliveiradosantos@gmail.com

${ }^{2}$ kleberkapelinski@hotmail.com

3 eduardosantos@unipampa.edu.br

${ }^{4}$ plinio@unipampa.edu.br
} 
Neste trabalho serão utilizados o PSO e o FA para resolver o problema de despacho econômico de carga considerando quinze unidades geradoras termoelétricas, perdas nas linhas de transmissão e limites máximos e mínimos de geração para cada geradora, tal como apresentado em [1], embora sem considerar zonas proibidas de operação. Em [1] os autores usam uma variação do PSO para resolver o DE. Para ambos algoritmos será utilizado um critério de parada considerando uma estimativa do erro nas 500 últimas iterações [10]. Por fim, os resultados obtidos serão comparados com a solução encontrada utilizando o MML.

Este trabalho está estruturado da seguinte forma: na Seção 2 é abordado o problema de Despacho Econômico de Carga (DE); na Seção 3 é apresentado o método de Otimização por Enxame de Partículas; na Seção 4 o método de Otimização por Enxame de Vagalumes; na Seção 5 são apresentados e discutidos os resultados obtidos na resolução do DE de um sistema com quinze termoelétricas. Por fim, na Seção 6, se encontram as principais conclusões e perspectivas futuras.

\section{Despacho Econômico de Carga}

Em um problema de DE com $n$ usinas termoelétricas, o custo total é dado pelo somatório do custo individual de cada unidade de geração, $\sum_{i=1}^{n} C_{i}\left(P_{i}\right)$, onde $C_{i}$ é a função de custo do combustível da unidade de geração $i$ em $\$ / \mathrm{h}$, que é aproximada por uma função quadrática convexa em termos da potência gerada [2], $C_{i}\left(P_{i}\right)=a_{1} P_{i}^{2}+b_{i} P_{i}+c_{i}$, e $P_{i}$ é a potência gerada pela mesma unidade $i$ em MW. Além disso, os limites de operação de cada usina são dados pelas restrições $P_{i}^{\min } \leq P_{i} \leq P_{i}^{\max }, i=1, \ldots, n$, e o somatório da potência gerada pelas unidades deve atender a demanda total do sistema:

$$
\sum_{i=1}^{n} P_{i}-P_{D}-P_{L}\left(P_{1}, \cdots, P_{n}\right)=0
$$

onde a perda nas linhas de transmissão é dada pela fórmula de perdas de Kron [5]:

$$
P_{L}\left(P_{1}, \cdots, P_{n}\right)=\sum_{i=1}^{n} \sum_{j=1}^{n} B_{i j} P_{i} P_{j}+\sum_{i=1}^{n} B_{0 i} P_{i}+B_{00},
$$

sendo $B_{i j}$ é o ij-ésimo elemento da matriz de coeficientes de perda simétrica, $B_{0 i}$ é o i-ésimo elemento do vetor de coeficiente de perdas e $B_{00}$ é a constante do coeficiente de perda. Matematicamente, o problema de despacho econômico apresentado pode ser escrito como o problema de otimização restrito não-linear dado pela equação (1):

$$
\begin{cases}\min & C\left(P_{1}, \cdots, P_{n}\right)=\sum_{i=1}^{n} C_{i}\left(P_{i}\right) \\ \text { s.a. } & \sum_{i=1}^{n} P_{i}-P_{D}-P_{L}\left(P_{1}, \cdots, P_{n}\right)=0 \\ & P_{i}^{\text {min }} \leq P_{i} \leq P_{i}^{\max }, i=1, \ldots, n\end{cases}
$$




\section{Otimização por Enxame de Partículas}

Em 1995, inspirado no comportamento social constatado em variadas espécies de pássaros, cardumes de peixes e enxames de insetos, a heurística Otimização por Enxame de Partículas (PSO) foi apresentada por James Kennedy e Russell Elberhart [4].

O PSO está baseado em uma população composta por indivíduos capazes de interagir entre si e com o meio ambiente. Portanto, cada partícula é influenciada por dois fatores: um social e outro cognitivo. O social, gBest $(\mathrm{gB})$, relaciona a influência que a melhor partícula exerce sobre toda população e o cognitivo, pBest $(\mathrm{pB})$, reflete o melhor resultado obtido individualmente [6].

Neste método, um enxame com $N$ partículas é inicializado em posições aleatórias dentro do espaço de busca. Em seguida, a função objetivo é calculada para cada partícula, e a partícula $i$, cuja posição é $\vec{x}_{i}$, deverá se mover com uma velocidade $\vec{v}_{i}$, a qual é calculada por (2):

$$
\vec{v}_{i}(k+1)=\vec{v}_{i}(k)+\varphi_{1} \epsilon_{1}\left(\vec{x}_{p_{B}}-\vec{x}_{i}(k)\right)+\varphi_{2} \epsilon_{2}\left(\vec{x}_{g_{B}}-\vec{x}_{i}(k)\right),
$$

sendo o índice $k$ o passo da iteração, $\vec{x}_{p_{B}}$ a posição onde ocorre o pBest, $\vec{x}_{g_{B}}$ a posição onde ocorre o gBest, $\epsilon_{1}, \epsilon_{2}$ variáveis aleatórias com distribuição uniforme no intervalo $[0,1]$ e as constantes $\varphi_{1}$ e $\varphi_{2}$ os parâmetros cognitivo e social, respectivamente. Assim, a posição da partícula $i$ é atualizada por (3):

$$
\vec{x}_{i}(k+1)=\vec{x}_{i}(k)+\vec{v}_{i}(k+1) .
$$

Como forma de evitar que as partículas não saiam do espaço de busca, são impostos limites para o módulo da velocidade das partículas, $v_{\max }>0$, conforme (4):

$$
\left|\vec{v}_{i}\right|>v_{\max } \Rightarrow \vec{v}_{i}=\frac{\overrightarrow{v_{i}}}{\left|\overrightarrow{v_{i}}\right|} v_{\max }
$$

\section{Otimização por Enxame de Vaga-lumes}

Existem muitas espécies de vaga-lumes, onde cada espécie tem uma forma particular de se comunicar através de sua luz. O ritmo, a frequência e quantia de tempo das piscadas definem o sistema de sinalização dos vaga-lumes [8]. Este sistema serve para atrair parceiros de acasalamento, atrair potenciais presas e, também serve como mecanismo de alerta da presença de predadores. A visibilidade de um vaga-lume está associada com a distância a que ele está do observador. Além disso, o ar também absorve uma parcela da luminosidade, o que se torna outro fator associado à visibilidade do vaga-lume.

$\mathrm{Na}$ otimização por enxame de vaga-lumes (FA) proposto em [8], a ideia é associar a função objetivo com a luz dos vaga-lumes, desta forma, quanto melhor a solução, o brilho do vaga-lume é maior. No algoritmo são idealizadas três regras básicas: todos vaga-lumes se atraem independente do gênero (são unissex); a atratividade de um vaga-lume está relacionada com o seu brilho (o menos brilhante tende a se dirigir ao mais brilhante); e o brilho do vaga-lume é afetado ou determinado pelo panorama da função objetivo.

Neste algoritmo existem dois problemas importantes, que são, a variação da intensidade de luz, e a formulação da atratividade. 
A atratividade de um vaga-lume é determinada por seu brilho. Em um caso simples, assume-se que a intensidade de luz de um vaga-lume é proporcional ao valor da função objetivo na sua posição. A atratividade $\beta$ é relativa, ela varia com a distância $r$ entre dois vaga-lumes $\left(r_{i j}\right)$. A intensidade de luz diminui quanto mais distante se estiver da fonte, e também a luz é absorvida no meio, sendo assim, a intensidade de luz $I$ varia com um grau de absorção.

De uma forma simples, pode-se aproximar a intensidade de luz $I$ a uma distância $r$ da fonte de luz $I_{s}$, pela expressão $I(r)=I_{s} e^{-\gamma r^{2}}$. Como a atração é proporcional à intensidade de luz vista pelos vaga-lumes, a atratividade é definida por $\beta(r)=\beta_{0} e^{-\gamma r^{2}}$, onde $\beta_{0}$ é a atratividade em $r=0$. Note que nestas expressões existe uma distância característica, onde a atratividade muda significativamente de $\beta_{0}$ para $\beta_{0} / e$. Esta distância característica é definida como $\Gamma=1 / \sqrt{\gamma}$.

A distância entre dois vaga-lumes é determinada pela distância Euclidiana, para $d$ dimensões do problema: $r_{i j}=\sqrt{\sum_{k=1}^{d}\left(\vec{x}_{i}^{k}-\vec{x}_{j}^{k}\right)^{2}}$. Desta forma, o movimento de um vaga-lume $i$, que é atraído por outro mais atrativo (mais brilhante) $j$, é determinado por:

$$
\vec{x}_{i}=\vec{x}_{i}+\beta_{0} e^{-\gamma r_{i j}^{2}}\left(\vec{x}_{j}-\vec{x}_{i}\right)+\alpha\left(\epsilon-\frac{1}{2}\right) .
$$

O segundo termo em (5) está relacionado à atração, e irá dirigir o vaga-lume $i$ para o $j$. O terceiro termo diz respeito ao comportamento aleatório do vaga-lume, onde $\alpha$ é a constante que dá peso a este comportamento e $\epsilon$ uma variável aleatória uniformemente distribuída no intervalo [0,1]. Em geral tem-se $\alpha \in[0,1]$ e $\beta_{0}=1$. O coeficiente de absorção $\gamma$ geralmente é constante e varia entre 0,01 e 100. Em [8] é mostrado que quando $\gamma \rightarrow 0$, o FA se reduz a um caso particular do PSO, onde todas as partículas conseguem ver todas as outras com precisão no espaço de busca.

\section{Resultados}

Nesta seção será resolvido o DE descrito por (1) para o sistema com quinze unidades geradoras apresentado em [1]. Neste sistema, $n=15$, a demanda de potência total é $P_{D}=2.630 \mathrm{MW}$ e todos os seus dados e parâmetro podem ser consultados em [1]. Foram considerados os seguintes parâmetros na implementação das heurísticas:

- PSO: foram realizadas 20 execuções considerando condições iniciais aleatórias e os parâmetros $N=100, \varphi_{1}=\varphi_{2}=2, v_{\max }=5$.

- FA: foram realizadas 20 execuções considerando condições iniciais aleatórias e os parâmetros $N=100, \alpha=0,5, \beta_{0}=\gamma=1$.

A função objetivo utilizada em ambas heurísticas é dada por:

$$
f\left(P_{1}, \ldots, P_{15}\right)=\sum_{i=1}^{15} C_{i}\left(P_{i}\right)+\phi\left|\sum_{i=1}^{15} P_{i}-P_{D}-P_{L}\left(P_{1}, \ldots, P_{15}\right)\right|
$$


onde o fator de penalização utilizado foi $\phi=50$. Além disso, como critério de parada foi calculado o erro relativo estimado nas últimas 500 iterações e verificado se esse erro é menor do que a toletância de $10^{-6}$ [10]. Todas as simulações computacionais foram realizadas utilizando o software Matlab executado em um computador com processador Intel(R) Core(TM) i3-3110M, de $2.40 \mathrm{GHz}$ e 4GB de RAM, sistema operacional Windows 8.1 Pro 64bits. Para o caso do FA, foi utilizada a implementação em Matlab disponível em $[9]$.

Na Tabela 1 são apresentadas estatísticas dos custos totais encontrados tando pelo PSO quanto pelo FA. Nesta tabela pode-se verificar que a melhor solução encontrada pelo FA, de $32.551,70 \$ / \mathrm{h}$, é aproximadamente $1,1 \%$ menor do que a apresentada pelo PSO, de $32.912,00 \$ / \mathrm{h}$. Além disso, o PSO apresentou uma variabilidade de soluções entre uma execução e outra significativamente maior do que o FA, conforme observado pelos valores de desvio padrão apresentados.

Tabela 1: Estatística dos Custos Totais

\begin{tabular}{|l|c|c|c|c|}
\hline & $\min (\$ / \mathrm{h})$ & média $(\$ / \mathrm{h})$ & $\max (\$ / \mathrm{h})$ & Desvio padrão $(\$ / \mathrm{h})$ \\
\hline PSO & $32.912,00$ & $33.108,61$ & $33.313,11$ & 109,95 \\
\hline FA & $32.551,70$ & $32.584,91$ & $32.686,94$ & 40,08 \\
\hline
\end{tabular}

Na Tabela 2 verifica-se que o custo computacional do FA (medido pelo número total de iterações) tende a ser bem maior do que o do PSO: em média foi 303,49\% maior.

Tabela 2: Estatística do Número Total de Iterações

\begin{tabular}{|l|c|c|c|c|}
\hline & min & média & $\max$ & Desvio padrão \\
\hline PSO & 1.000 & 2.125 & 4.500 & 1.266 \\
\hline FA & 1.000 & 8.650 & 22.000 & 7.025 \\
\hline
\end{tabular}

Na Tabela 3 estão os melhores resultados obtidos pelo PSO e pelo FA, assim como a solução obtida via método de multiplicadores de Lagrange, para comparação. A solução via MML foi obtida usando o comando nativo fsolve do Matlab para resolver numericamente o sistema de equações não-lineares associado. Embora o FA seja mais custoso computacionalmente, apresentando um número total de iterações $550 \%$ maior do que o PSO, seu melhor resultado obtido é bem mais próximo da solução do MML $(0,01 \%)$ do que o PSO $(1,12 \%)$. Por fim, na Figura 1 são apresentados os gráficos de convergência da melhor solução tanto do FA, quanto do PSO, em comparação com a solução obtida via MML.

\section{Conclusões}

Neste trabalho aplicou-se as heurísticas de otimização por enxames de partículas (PSO) e vaga-lumes (FA) na resolução de um problema de despacho econômico de carga considerando quinze unidades geradoras termoelétricas, perdas nas linhas de transmissão e limites 
Tabela 3: Melhores Resultados e Comparação com MML

\begin{tabular}{|l|c|c|c|}
\hline Saídas de Potência & MML & PSO & FA \\
\hline Unidade 1(MW) & 455,0000 & 335,7774 & 454,9984 \\
\hline Unidade 2(MW) & 455,0000 & 454,9628 & 455,0000 \\
\hline Unidade 3(MW) & 130,0000 & 74,7213 & 129,9999 \\
\hline Unidade 4(MW) & 130,0000 & 97,6500 & 130,0000 \\
\hline Unidade 5(MW) & 238,5672 & 425,1476 & 256,5043 \\
\hline Unidade 6(MW) & 460,0000 & 459,1562 & 460,0000 \\
\hline Unidade 7(MW) & 465,0000 & 421,9807 & 465.0000 \\
\hline Unidade 8(MW) & 60,0000 & 76,5381 & 60.0000 \\
\hline Unidade 9(MW) & 25,0000 & 70.2370 & 25,0749 \\
\hline Unidade 10(MW) & 25,7775 & 72,1925 & 39,3442 \\
\hline Unidade 11(MW) & 77,5615 & 73,2532 & 54,8060 \\
\hline Unidade 12(MW) & 80,0000 & 30,6467 & 71,7712 \\
\hline Unidade 13(MW) & 25,0000 & 25,2195 & 25,0004 \\
\hline Unidade 14(MW) & 15,0000 & 24,6739 & 15,0009 \\
\hline Unidade 15(MW) & 15,0000 & 24,0569 & 15,0021 \\
\hline$P_{L}(\mathrm{MW})$ & 26,9062 & 36,2138 & 27,5023 \\
\hline$P_{D}(\mathrm{MW})$ & $2.630,0000$ & $2.630,0000$ & $2.630,0000$ \\
\hline$\sum P_{i}(\mathrm{MW})$ & $2.656,9062$ & $2.666,2138$ & $2.657,5023$ \\
\hline Custo(\$/h) & $32.547,50$ & $32.912,00$ & $32.551,70$ \\
\hline Iterações & - & 2.000 & 13.000 \\
\hline Tempo (seg) & - & 8,3523 & 367,4753 \\
\hline
\end{tabular}

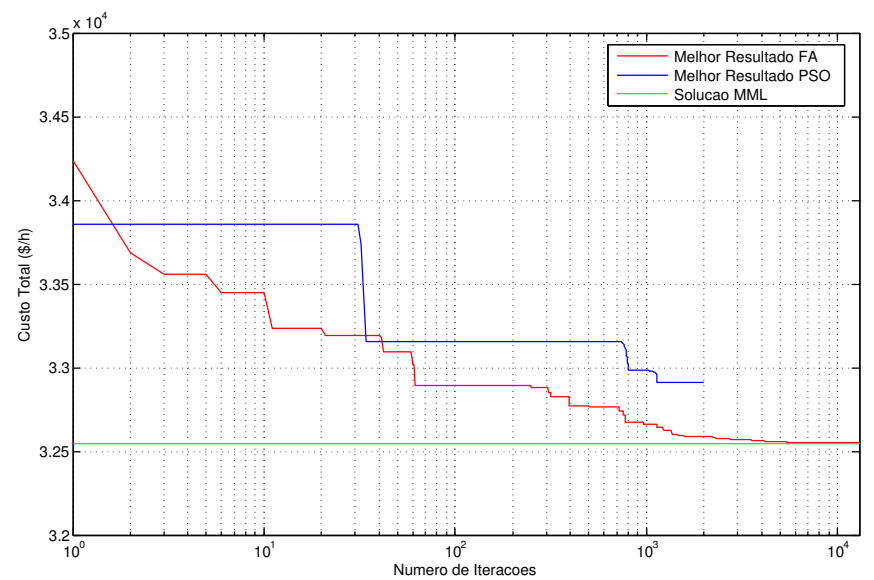

Figura 1: Convergência das Melhores Soluções do FA e PSO.

máximos e mínimos de geração para cada geradora. Além disso, comparou-se a eficácia e 
eficiência destes dois métodos entre si, assim como com o resultado obtido através de sua resolução via multiplicadores de Lagrange.

A principal conclusão obtida com as simulações computacionais realizadas foi a de que, embora o PSO tenha se mostrado um método mais eficiente, apresentando convergência mais rápida com o critério de parada adotado, o FA foi mais eficaz, no sentido de que obteve uma solução melhor do que a do PSO. Enquando o PSO obteve um custo mínimo de $32.912,00 \$ /$ h em 2.000 iterações (8, 3523 segundos), o FA obteve um custo de $32.551,70$ $\$ /$ h em 13.000 iterações $(367,4753$ segundos). Comparando com o custo ótimo obtido via MML, de $32.547,50 \$ / \mathrm{h}$, o FA apresentou um custo apenas $0,01 \%$ maior do que ele, enquanto o custo via PSO foi de $1,12 \%$ maior.

Como continuidade a este trabalho, serão estudadas formas de tornar o algoritmo dos vaga-lumes mais eficiente computacionalmente.

\section{Referências}

[1] L. S. Coelho, C. S. Lee. Solving economic load dispatch problems in power systems using chaotic and gaussian particle swarm optimization approaches. International Journal of Electrical Power E Energy Systems, Elsevier v. 30, n. 5, p. 297-307, 2008.

[2] M. Djurovic, A. Milancic, M. Krsulja. A simplied model of quadratic cost function for thermal generators, Proceedings of the 23rd DAAAM Symposium, p. 25-25, 2012.

[3] A. Gómez-Expósito, A. Conejo, C. Cañizares, Sistemas de Energia Elétrica: análise e operação, LTC, RJ, 2011.

[4] A. Mahor, V. Prasad, S. Rangnekar. Economic dispatch using particle swarm optimization:a review. Renewable and sustainable energy reviews, Elsevier v. 13, n. 8, p. 2134-2141, 2009.

[5] H. Saadat, Power System Analysis, McGraw-Hill, 1999.

[6] A. B. S. Serapião. Fundamentos de Otimização por Inteligência de Enxames:uma visão geral. Controle e Automação, SBA: Sociedade Brasileira de Automática, v. 20, n. 3, p. 271-304, 2009 .

[7] C. P. Simon, L. Blume, Matemática para Economistas, Bookman, 2004.

[8] X. S. Yang. Firefly algorithms for multimodal optimization. International symposium on stochastic algorithms, Springer, p. 169-178, 2009.

[9] X. S. Yang, Firefly Algorithm, version 1.2, (https://www.mathworks.com/ matlabcentral/fileexchange/29693-firefly-algorithm), MATLAB Central File Exchange, acessado em 2 de março de 2017.

[10] K. Zielinski, D. Peters, R. Laur. Stopping Criteria for Single-Objective Optimization. Proceedings of the Third International Conference on Computational Intelligence, Robotics and Autonomous Systems, Citeseer of 2005. 